\title{
CLASSIFICATION OF RELATIVE MINIMA SINGULARITIES
}

\author{
ALEKSEI A. DAVYDOV \\ Vladimir State University \\ Gorkiu str. 87, 600026 Vladimir, Russia \\ E-mail: davydov-m2@vpti.vladimir.su \\ VLADIMIR M. ZAKALYUKIN \\ Moscow Aviation Institute \\ Volokolamskoe sh. 4, 125871 Moscow, Russia \\ E-mail:vladimir@zakal.mccme.ru
}

1. Introduction. The following elementary extremal problem with constraints arises as a base model for various settings in parametric optimisation theory:

Let $g: N^{n} \rightarrow M^{m}$ be a proper smooth mapping of smooth manifolds $N^{n}$ and $M^{m}$ and let $f: N^{n} \rightarrow \mathbf{R}$ be a smooth function on $N^{n}$.

The function

$$
F(q)=\min \{f(p) \mid p \in N, g(p)=q\}, q \in g(N) \subset M,
$$

defined on the image of $g$, is called the relative minima function. It provides a solution of the extremal problem $f(\cdot) \rightarrow$ min under the constraint $g(\cdot)=q$, where $q \in M$.

We call $g$ the constraint mapping, $M$ the parameter manifold, $f$ the minimising function and the mapping $(f, g): N \rightarrow \mathbf{R} \times M$ the pair-mapping.

Only the case $n \geq m$, when the relative minima function might be defined on the subset of full dimension $m$, will be considered.

Relative minima function is, generally speaking, neither smooth nor continuous. Its singularities describe those of the boundary of attainability domains (for given time) of control systems, propagations of wave fronts, singularities of solutions of parameter depending extremal problems (for example, that of production of optimal mixtures) and so on.

\footnotetext{
1991 Mathematics Subject Classification: 58, 49.

Partially supported by RFBI 960100710, 970100713 and INTAS 960713 grants.

The paper is in final form and no version of it will be published elsewhere.
} 
Here we continue the study of the generic singularities of relative minima function started in [5], [6]. We present complete lists of these singularities when the dimension $m$ of parameter is 3 and 4 up to the following equivalence relation: two germs of relative minima function are called $\Gamma$-equivalent if their graphs might be mapped one to the other by a germ of smooth diffeomorphisms of the product of the parameter space by a line which preserves the projection onto the parameter.

The inverse image under the generic pair-mapping of a point $P$ on the graph of relative minima consists of at most finite number of points $X_{1}, \ldots, X_{k}$. Near $P$ this graph coincides with the graph of the minimum of finite number of local relative minima functions, defined by the germs of pair-mappings at these preimages $X_{1}, \ldots, X_{k}$. The singularities of local relative minima function are called point singularities. Thus, lists of normal form of singularities consist of admissible combinations of point singularities.

We classify also all simple (in the singularity theory sense) stable singularities and determine the nice dimensions, for which all generic singularities are stable and simple.

The 3-dimensional parameter belongs to this nice dimension region (Theorem 2). There exist 34 different types of generic singularities when $m=3$. But only 24 of them appear when $n>4$, and the list remains the same for all such $n$ due to a certain stabilisation property of singularities.

The lists of normal forms provide, of course, the diffeomorphic types of singularities of domain, where relative minima function is defined, as well as the sets of it discontinuity.

The proofs of the classification theorems are based on the study of the diagrams of mappings. According to the classical result of J. Mather [1] the classification of stable (with respect to right-left equivalence) mappings reduces to enumeration of versal deformation of contact singularities. Similarly, the classification of stable diagrams, corresponding to relative minima singularities, might be reduced to the study of versal deformations of the contact of smooth submanifold (the graph of pair-mapping) with the coordinate flag of two subspaces.

This approach clarifies the close interconnections with the singularities of functions on the manifold with boundary [2], [9], discriminants of simple projections [8], singularities of diagrams of mappings and of contact with flags [12].

The constraint mapping being a submersion, the problem becomes that of the singularities of minima of parameter depending functions. The latter was widely studied (see for example [3], [10]).

Time-optimisation of a system with smooth strictly convex indicatrices give rise to a special class of relative minima singularities, related to the singularities of families of wave fronts [2], [11]. They are briefly discussed in the following section.

Note finally that reversing the sign of minimising function one gets the corresponding results on relative maxima.

2. Definitions and results. A pair from an open and dense subset of the space of pairs, equipped with Whitney $C^{\infty}$-fine topology, will be called a generic one. A germ of relative minima functions is called stable, if for any nearby pair a germ of relative minima function at some nearby point in parameter space is $\Gamma$-equivalent to the initial 
one. A germ of relative minima functions is called simple, if for any nearby pair all germs of relative minima functions at nearby points in parameter space belong to a finite number of equivalence classes.

Dimensions $n$ and $m$ are called nice if all germs of relative minima function of generic pair-mappings $(f, g): N^{n} \rightarrow \mathbf{R} \times M^{m}$ are simple.

THEOREM 1. The dimensions $n=m$ are nice if and only if $m<6$. The dimensions $n>m$ are nice if and only if $m<4$.

THEOREM 2. Let the dimension of parameter be $m=3$. Then a relative minima function germ of a generic pair at an arbitrary point is stable, simple and is $\Gamma$-equivalent to a germ at zero of one of the following functions from the second column of Table 1 , provided that

- if $n \geq 4$, it should be one of the first 24 functions of the table;

- if $n=4$, it should be one of the first 27 ones;

- if $n=3$, it should be any function of the table, except 24th, 25th, 26th and 27th.

Notes. Third column of the table contains values of codimension in $\mathbf{R}^{3}$ of the stratum of given relative minima function singularity.

Local coordinates on the parameter space are denoted by $x, y, z$.

Dimensions $n$ intrinsic for given singularity are shown in fourth column.

TABle 1

\begin{tabular}{|r|c|c|c|}
\hline No & Normal form & codim & $\operatorname{dim} N$ \\
\hline 1 & 0 & 0 & $\geq 3$ \\
2 & $-|x|$ & 1 & $\geq 3$ \\
3 & $\min \{-|x|, y\}$ & 2 & $\geq 3$ \\
4 & $\min \{-|x|, y, z\}$ & 3 & $\geq 3$ \\
5 & $-\sqrt{x}$ & 1 & $\geq 3$ \\
6 & $\min \{-\sqrt{x}, 1\}$ & 1 & $\geq 3$ \\
7 & $\min \{-\sqrt{x},-|y|+1\}$ & 2 & $\geq 3$ \\
8 & $\min \{-\sqrt{x}, y\}$ & 2 & $\geq 3$ \\
9 & $\min \{-\sqrt{x},-|y|+z\}$ & 3 & $\geq 3$ \\
10 & $\min \{-\sqrt{x}, \min \{-|y|, z\}+1\}$ & 3 & $\geq 3$ \\
11 & $\min \{-\sqrt{x},-\sqrt{y}+1\}$ & 2 & $\geq 3$ \\
12 & $\min \{-\sqrt{x},-\sqrt{y}+1,2\}$ & 3 & $\geq 3$ \\
13 & $\min \{-\sqrt{x},-\sqrt{y}+z\}$ & 3 & $\geq 3$ \\
14 & $\min \{-\sqrt{x},-\sqrt{y}+z, 1\}$ & 3 & $\geq 3$ \\
15 & $\min \{-\sqrt{x},-\sqrt{y}+1,-|z|+2\}$ & 3 & $\geq 3$ \\
16 & $\min \{-\sqrt{x}, \min \{-\sqrt{y}, z\}+1\}$ & 3 & $\geq 3$ \\
17 & $\min \{-\sqrt{x},-\sqrt{y}+1,-\sqrt{z}+2\}$ & 3 & $\geq 3$ \\
18 & $\min \{-\sqrt{x},-\sqrt{y}+1,-\sqrt{z}+2,3\}$ & 2 & $\geq 3$ \\
19 & $\min \left\{w \mid w^{3}+x w+y=0\right\}$ & 3 & $\geq 3$ \\
20 & $\min \left\{\min \left\{w \mid w^{3}+x w^{2}+y=0\right\}, z\right\}$ & & \\
21 & $\min \left\{-\sqrt{z}, \min \left\{w \mid w^{3}+x w+y=0\right\}+1\right\}$ & 3 \\
\hline
\end{tabular}


TABLE 1 (continued)

\begin{tabular}{|r|c|c|c|}
\hline No & Normal form & codim & $\operatorname{dim} N$ \\
\hline 22 & $\min \left\{w \mid w^{4}+x w^{2}+y w+z=0\right\}$ & 3 & $\geq 3$ \\
23 & $\min \left\{\min \left\{w \mid w^{4}+x w^{2}+y w+z=0\right\}, 1\right\}$ & 3 & $\geq 3$ \\
24 & $\min \left\{w^{4}+x w^{2}+y w \mid w \in R\right\}$ & 3 & $\geq 4$ \\
25 & $\min \left\{u^{2}+w^{2}+u x+w y \mid u^{2}-w^{2}=z\right\}$ & 3 & $=4$ \\
26 & $\min \left\{u w+u x+w y \mid u^{2}+w^{2}=z\right\}$ & 3 & $=4$ \\
27 & $\min \left\{\min \left\{u w+u x+y w \mid u^{2}+w^{2}=z\right\}, 1\right\}$ & 3 & $=4$ \\
28 & $\min \{-|y| \sqrt{x}, z\}$ & 3 & $=3$ \\
29 & $\min \{-|y| \sqrt{x},-\sqrt{z} \pm 1\}$ & 3 & $=3$ \\
30 & $\min \{-|y| \sqrt{x},-\sqrt{z} \pm 1,2\}$ & 3 & $=3$ \\
31 & $-|y| \sqrt{x}$ & 2 & $=3$ \\
32 & $\min \left\{ \pm w^{2} \mid w^{3}+x w^{2}+y w+z=0\right\}$ & 2 & $=3$ \\
33 & $\min \{-|y| \sqrt{x}, 1\}$ & 3 & $=3$ \\
34 & $\min \{-|y| \sqrt{x},-|z|+1\}$ & & 3 \\
\hline
\end{tabular}

TheOREM 3. Let the dimension of parameter be $m=4$. Then a point singularity of relative minima function germ of a generic pair at an arbitrary point is $\mathbf{R}^{+}$-equivalent to a germ at the origin of one of the following functions (from the second column of Table 2):

- $B_{k, 1}(1 \leq k \leq 5), B_{k, 2}(2 \leq k \leq 4), B_{3,3}, X_{4}^{ \pm}$, if $n=4$;

$-B_{k, 1}(1 \leq k \leq 5), A_{3}, A_{5}, C_{2,2}^{ \pm}, F_{5}, F_{5}^{*}$, if $n=5$;

$-B_{k, 1}(1 \leq k \leq 5), A_{3}, A_{5}, F_{5}^{*}, M_{6}^{ \pm}$, if $n=6$;

$-B_{k, 1}(1 \leq k \leq 5), A_{3}, A_{5}, F_{5}^{*}$, if $n>6$.

Notes. Local coordinates on the parameter space are denoted by $q_{1}, \ldots, q_{4}$.

Dimensions $n$ intrinsic for given singularity and the codimension of its stratum in parameter space are given below the normal form.

The functional moduli $\lambda, \mu, \nu$ are arbitrary functions in variables $q_{1}, q_{2}, q_{3}$.

Re mark. If $m=4$, a relative minima function germ of a generic pair at any point is $\Gamma$-equivalent either to the point singularities listed above or to the minimum of the collection of those whose total codimension does not exceed $n$.

TABLE 2

\begin{tabular}{|c|c|}
\hline Notation & Normal form \\
\hline$A_{3}$ & $\min \left\{x^{4}+q_{1} x^{2}+q_{2} x \mid x \in R\right\}$ \\
& $\operatorname{codim}=2, n>4$ \\
\hline$A_{5}$ & $\min \left\{x^{6}+q_{1} x^{4}+q_{2} x^{3}+q_{3} x^{2}+q_{4} x \mid x \in R\right\}$ \\
& $\operatorname{codim}=4, n \geq 5$ \\
\hline$B_{k, 1}$ & $\min \left\{x \mid x^{k}+q_{1} x^{k-2}+\ldots+q_{k-2} x+q_{k-1}=0\right\}$ \\
$1 \leq k \leq 5$ & $\operatorname{codim}=k-1, n \geq 4$ \\
\hline$B_{k, 2}$ & $\min \left\{ \pm x^{2} \mid x^{k}+q_{1} x^{k-1}+\ldots+q_{k-1} x+q_{k}=0\right\}$ \\
$2 \leq k \leq 4$ & $\operatorname{codim}=k, n=4$ \\
\hline
\end{tabular}


TABLE 2 (continued)

\begin{tabular}{|c|c|}
\hline Notation & Normal form \\
\hline$B_{3,3}$ & $\min \left\{x^{3}+x q_{4} \mid x^{3}+q_{1} x^{2}+q_{2} x+q_{3}=0\right\}$ \\
& $\operatorname{codim}=4, n=4$ \\
\hline$X_{4}^{ \pm}$ & $\min \left\{x+2 y \mid x y+q_{1}=x^{2} \pm y^{2}+x q_{2}+y q_{3}+q_{4}=0\right\}$ \\
& $\operatorname{codim}=4, n=4$ \\
\hline$C_{2,2}^{-}$ & $\min \left\{x^{2}+y^{2}+q_{2} x+q_{3} y \mid x y=q_{1}\right\}$ \\
& $\operatorname{codim}=4, n=5$ \\
\hline$C_{2,2}^{+}$ & $\min \left\{x y+q_{2} x+q_{3} y \mid x^{2}+y^{2}=q_{1}\right\}$ \\
& $\operatorname{codim}=4, n=5$ \\
\hline$F_{5}$ & $\min \left\{y^{2}+x q_{4} \mid x^{2}+y^{3}+q_{1} y^{2}+q_{2} y+q_{3}=0\right\}$ \\
& $\operatorname{codim}=4, n=5$ \\
\hline$F_{5}^{*}$ & $\min \left\{x+y^{2} \lambda(q) \mid \begin{array}{c}\left.x^{2} \pm y^{4}+q_{1} y^{3}+q_{2} y^{2}+q_{3} y+q_{4}=0\right\} \\
\operatorname{codim}=4, n \geq 5\end{array}\right.$ \\
\hline$Z_{6}^{e}$ & $\min \left\{ \pm x^{2}+\mu(q) y^{2}+q_{1} x+q_{2} y+x_{3} z \mid x^{2}+y^{2}+z^{2}=q_{4}\right\}$ \\
$\mu(0) \neq 0, \operatorname{codim}=4, n=6$
\end{tabular}

Remark. Let $W_{t} \subset M$ be a time dependent family of wave fronts. For a point $q \in M$ the minimal of values of time $t$ such that $q \in W_{t}$ is a value of a corresponding relative minima function. The singularities of these functions are special. Suppose the big front $\widetilde{W}=\left(t, W_{t}\right) \subset \mathbf{R} \times M$ in a space-time is a projection of a smooth Legendrian submanifold in $P T^{*}(\mathbf{R} \times M)$. Then the corresponding minimising function is regular and the constraint mapping has corank at most one. In particular, the classes of Theorems 2 and 3 satisfying these conditions form all minimal singularities [11] of envelopes and caustics of families of wave-fronts in $\mathbf{R}^{3}$ and $\mathbf{R}^{4}$.

3. General constructions. Consider the diagram

$$
N \stackrel{(f, g)}{\longrightarrow} \mathbf{R} \times M \stackrel{\pi}{\longrightarrow} M, \quad x \mapsto(f(x), g(x)) \mapsto g(x),
$$

associated to a pair-mapping $(f, g)$. Here $\pi$ is the natural projection.

Sets of three diffeomorphisms $\left(\theta_{1}, \theta_{2}, \theta_{3}\right)$, generating a commutative diagram

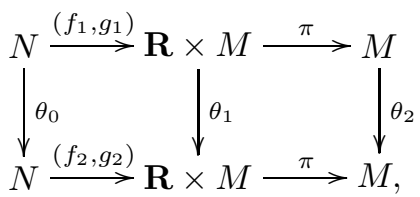

whose rows correspond to two pair-mappings, form a group, which we denote by $\Gamma$. This equivalence group $\Gamma$ acts on the space of pair-mappings (via the associated row-diagrams).

Denote by $\Gamma^{+}$the subgroup of $\Gamma$, preserving the orientation of the fibres of the projection $\pi$. Obviously the relative minima functions of two $\Gamma^{+}$-equivalent diagrams are $\Gamma$-equivalent. 
Denote by $R^{+}$the subgroup of $\Gamma^{+}$equivalences for which the diffeomorphism $\theta_{1}$ is simply a shift along each fibre of the projection $\pi$,

$$
\theta_{1}:(t, q) \rightarrow(t+\phi(q), Q(q)), \quad t \in \mathbf{R}, q \in M^{m} .
$$

Classification of $\Gamma^{+}$-stable pairs plays an important role in the proofs of Theorems $1-3$. In particular, standard arguments of singularity theory imply the following infinitesimal criterion for $\Gamma^{+}$-stability of the germ (or multigerm) of the pair-mapping $(f, g): \mathbf{R}^{n} \rightarrow$ $\mathbf{R} \times \mathbf{R}^{m},(f, g):(0,0) \mapsto(0,0)$ at the origin.

Proposition 1. The germ at the origin of the pair $(f, g)$, such that $f(0)=0$ and $g(0)=0$, is $\Gamma^{+}$-stable, if and only if for any germ of the pair-mapping $(\phi, \psi):(N, 0) \rightarrow$ $\mathbf{R} \times \mathbf{R}^{m}$ there exist a germ at $(0,0) \in \mathbf{R} \times \mathbf{R}^{m}$ of the mapping $\theta: \mathbf{R} \times \mathbf{R}^{m} \rightarrow \mathbf{R} \times \mathbf{R}^{m}$, preserving the projection $\pi: \mathbf{R} \times \mathbf{R}^{m} \rightarrow \mathbf{R}^{m}, \theta:(t, q) \mapsto(\alpha(t, q), \beta(q))$, and a germ of a vector field $v$ on $N$ such that (below $i_{v} d$ denotes the derivative along $v$ ):

$$
\left\{\begin{array}{l}
\phi=\alpha \circ(f, g)+i_{v} d f \\
\psi=\beta \circ g+i_{v} d g .
\end{array}\right.
$$

Remark. The corresponding infinitesimal stability criterion for the $R^{+}$group requires the additional condition: the function $\alpha$ should be the function only in $q$ variables.

The left-hand side in these formulas contains modules of composed functions $\beta \circ(f, g)$ over the ring of germs of functions on the target space, which are not so easy to operate with. The well known Mather-Martinet theorem [1] simplifies the analogical difficulty for the case of right-left stability of smooth mappings. The infinitesimal stability criterion happens to be equivalent to the contact versality of the extended deformation of the corresponding graph mapping, which deals only with modules over the ring of functions on the source space.

Describe now the generalisation of this classical theorem to the case of pair-mappings (relating them to the singularities of the submanifold contact with non-complete flags $[12])$.

To a pair-mapping $(f, g)$ associate also the graph mapping

$$
G_{f, g}: N \rightarrow N \times \mathbf{R} \times \mathbf{R}^{m}, \quad G: x \mapsto(x, f(x), g(x)) .
$$

Consider the flag consisting of two subspaces $K_{1} \supset K_{2}$,

$$
K_{1}=N \times R \times\{0\} ; \quad K_{2}=N \times\{0\} \times\{0\},
$$

in the target space $K_{0}=N \times \mathbf{R} \times \mathbf{R}^{m}$ of the graph mapping. The shift diffeomorphism $S: K_{0} \rightarrow K_{0}, S:(x, f, g) \mapsto\left(x, f-f\left(x_{0}\right), g-g\left(x_{0}\right)\right)$ of the pair-mapping graph sends its distinguished point $\left(x_{0}, f\left(x_{0}\right), g\left(x_{0}\right)\right)$ to a point in $K_{2}$.

Two pair-mapping germs will be called flag-contact equivalent, if, after corresponding shifts of the distinguished points, the graph of one of them might be sent to the other by a diffeomorphism $\Theta: K_{0} \rightarrow K_{0}$ which preserve the flag $\Theta\left(K_{1}\right)=K_{1}, \Theta\left(K_{2}\right)=K_{2}$.

Obviously the $\Gamma^{+}$-equivalent pair-germs are flag-contact equivalent: $\Theta=\left(\theta_{0}, \theta_{1}\right)$.

The classes of flag-contact orbits of the germs of the graphs form a stratification of the space of germs of pair-mappings. The appearance of a given flag-contact class as the generic singularity of pair-mapping depends on the codimension of this class in the space 
of pair-mapping germs with zero-value at the distinguished point (whether it exceeds $n$ or not). Just this codimension is considered below.

For a graph-mapping $G_{f, g}$ define its shift-deformation $G_{f, g}(\lambda)$ with $m+1$ additive parameters $\lambda_{0}, \lambda=\left(\lambda_{1}, \ldots, \lambda_{m}\right)$ as follows:

$$
G_{f, g}(\lambda):(x, \lambda) \mapsto\left(x, f(x)+\lambda_{0}, g(x)+\lambda\right) .
$$

The flag-contact equivalence is J. Damon's ([4]) geometrical subgroup of A-equivalencies of graph mappings, and the natural notion of flag contact versality of a deformation is equivalent to the corresponding infinitesimal versality. For shift-deformation this infinitesimal versality has the following form.

Denote by $C_{N}$ the ring of germs at the origin of the function on $N$, and by $I_{g}$ the ideal in $C_{N}$, generated by the components of the mapping $g$. Denote by $P$ the $C_{N}$-module of pair-germs at the origin, and by $K_{f, g}$ submodule of $P$ formed by pair-germs $(\tilde{\varphi}, \tilde{\psi})$, whose $\tilde{\psi}$-components belong to $I_{g}$ and whose $\tilde{\varphi}$-component belongs to the ideal $I_{f, g}$, generated by the components of pair-mapping.

Let $W_{f, g}$ be a submodule of $P$, formed by the pair-mappings of the form $i_{v} d(f, g)$, where $v$ is the germ at the origin of a vector field on $N$. Let, finally, $D_{g}$ be a submodule of $K_{f, g}$ formed by the pair-mapping germs, whose all components belong to $I_{g}$.

Proposition 2. The germ of the deformation $G_{f, g}(\lambda)$ is flag-contact infinitesimally versal if and only if for any germ of the pair-mapping

$$
(\varphi, \psi):(N, 0) \rightarrow \mathbf{R} \times \mathbf{R}^{m+1}, \quad \alpha: x \mapsto\left(\alpha_{1}, a\right)
$$

there exists a pair-germ $(\tilde{\varphi}, \tilde{\psi})$, whose $\tilde{\psi}$-components belong to $I_{g}$ and whose $\tilde{\varphi}$-component belongs to the ideal $I_{f, g}$, generated by the components of pair-mapping, there exist a germ of a vector field $v$ on $N$ and a set of constants $a_{0}, a=\left(a_{1}, \ldots, a_{m}\right)$ such that

$$
\left\{\begin{array}{l}
\phi=\tilde{\phi}+i_{v} d f+a_{0} \\
\psi=\tilde{\psi}+i_{v} d g+a
\end{array}\right.
$$

In other words, the factor module $P /\left\{K_{f, g}+W_{f, g}\right\}$ is generated over $\mathbf{R}$ by constant mappings.

The versal shift-deformation will be called strictly versal if the ideal $I_{f, g}$ in the previous proposition might be substituted by the sum of $I_{g}$ and a $\mathbf{R}$-module generated by certain finite collection of powers of the function $f$. This is equivalent to the additional claim that some power of $f$ belongs to the ideal $I_{g}$.

Proposition 3.

(i) If a germ (multigerm) of the pair-mapping is $\Gamma^{+}$-stable then its shift-deformation is versal (with respect to flag-contact equivalences).

(ii) The strict infinitesimal versality of shift-deformation $G_{f, g}(\lambda)$ implies $\Gamma^{+}$-stability of the germ $(f, g)$.

Proof. The first statement is evident, since the infinitesimal $\Gamma^{+}$-stability implies infinitesimal shift-versality. To prove the second part, consider the $C_{N}$-module $A=P / W_{f, g}$. The strict infinitesimal versality of $G$ implies that the factor module $A / I_{g} A$ is generated 
over $\mathbf{R}$ by the constants in each row and a finite set of powers of $f$ in the first one. According to the Malgrange preparation theorem module $A$ is a finitely generated module over the ring $C_{M}$ of germs of composed functions $y \circ g$ with the same system of generators. Thus for every pair $(\varphi, \psi)$ there exists a decomposition

$$
\left\{\begin{array}{l}
\varphi=\varphi_{0}(g)+\ldots+\varphi_{k}(g) f^{k}+i_{v} d f \\
\psi=\tilde{\psi}(g)+i_{v} d g
\end{array}\right.
$$

with certain germs of functions $\varphi_{i}$ defined on and a mapping $\tilde{\psi}: M, 0 \rightarrow M, 0$. This decomposition coincides with that of the $\Gamma^{+}$-stability criterion.

Let the constraint mapping germ $(g, 0)$ have rank $r$. By choosing appropriate local coordinates $x, y$ on $N, 0, x \in \mathbf{R}^{n-r}, y \in \mathbf{R}^{r}$ and $z, u$ on $M, 0, z \in \mathbf{R}^{m-r}, u \in \mathbf{R}^{r}$, the mapping takes the form $g:(x, y) \mapsto(z(x, y), u)$, where $u=y$ and the components $z_{i}(x, 0)$ belong to the square of the maximal ideal of the ring $C_{x}$ of germs at the origin of functions in $x$.

The mapping germ $z: \mathbf{R}^{n-r} \rightarrow \mathbf{R}^{m-r}, z: x \mapsto z(x, 0)$ is called the genotype of the mapping germ $g$. Right-left stable mapping germs are classified by the contact classes of their genotypes.

The genotype of a pair-mapping germ $(f, g)$ is a pair-mapping germ $(w, z): \mathbf{R}^{n-r} \rightarrow$ $\mathbf{R} \times \mathbf{R}^{m-r}$, where $z$ is the genotype of the constraint mapping germ and function $w(x)=$ $\left.f(x, y)\right|_{y=0}$. Define $\xi_{i}(x)=\left.\frac{\partial f}{\partial y_{i}}\right|_{y=0}$ and $\chi_{i}(y)=\left.\frac{\partial g}{\partial y_{i}}\right|_{y=0}$. The pair-mapping germ $(f, g)$ is called geno-versal if for any germ of the pair-mapping

$$
(\varphi, \psi):\left(\mathbf{R}^{n-r}, 0\right) \rightarrow \mathbf{R} \times \mathbf{R}^{m-r}
$$

there exist:

- a pair-germ $(\tilde{\varphi}, \tilde{\psi})$, whose $\tilde{\psi}$-components belong to $I_{z}$, generated by the components of the genotype of the constraint mapping, and $\tilde{\varphi}$-component belongs to the ideal $I_{w, z}$, generated by the components of the pair-mapping genotype,

- a germ of a vector field $v$ on $\mathbf{R}^{n-r}$ and a set of constants $a_{0}, a=\left(a_{1}, \ldots, a_{m}\right), b_{j}$, $j=1, \ldots, r$, such that

$$
\left\{\begin{array}{l}
\varphi=\tilde{\varphi}+i_{v} d f+a_{0}+\xi_{1} b_{1}+\ldots+\xi_{r} b_{r} \\
\psi=\tilde{\psi}+i_{v} d g+a+\chi_{1} b_{1}+\ldots+\chi_{r} b_{r} .
\end{array}\right.
$$

The geno-versal pair-germ will be called strictly geno-versal if certain power of $w$ belongs to the ideal $I_{z}+W_{w}$.

Malgrange's preparation theorem and the above infinitesimal stability criteria imply

Proposition 4.

(i) If the germ of pair-mapping is $\Gamma^{+}$-stable then it is geno-versal.

(ii) If $(f, g)$ is strictly geno-versal then it is $\Gamma^{+}$-stable.

Remarks.

1. The $R^{+}$-stability is equivalent to $\Gamma^{+}$-stability with the additional condition on the above decomposition: $\tilde{\varphi} \in I_{z}$.

2. If the pair-germ $(f, g)$ is weighted homogeneous then $w \in J$ and geno-versality implies strict geno-versality and even $R^{+}$-geno-versality. 
3. A $\Gamma^{+}$-stable pair-germ $(f, g)$ with given genotype $(w, z)$ is $\Gamma^{+}$-equivalent to a standard versal unfolding $(F, G)$ of the genotype, which is affine in $y$-variables and is defined as follows

$$
\begin{gathered}
F(x, y)=w(x)+\xi_{1}(x) y_{1}+\ldots+h \xi_{r}(x) y_{r}, \\
G(x, y)=\left(\begin{array}{c}
z(x)+\chi_{1} y_{1}+\ldots+\chi_{r} y_{r} \\
y
\end{array}\right)
\end{gathered}
$$

where pair-mappings $\left(\xi_{i}, \chi_{i}\right), i=1, \ldots, r$, pair-mappings of the form $\left(w^{j}, 0\right), j=1,2, \ldots$ and constant mappings form a system (may be not minimal) of generators of the factor module $P_{x} /\left(W_{w, z}+D_{z}\right)$.

4. Flag-contact simple pairs. The stable pair-germ is called simple with respect to certain equivalence group (for example, $\Gamma^{+}$or flag-contact) if it has a representative, whose germs at all nearby points belong to a finite number of equivalence classes. Since $\Gamma^{+}$-equivalence of two pair-germs implies flag-contact equivalence of them, a $\Gamma^{+}$-simple germ is flag-contact simple as well. This means that some neighbourhood of its genotype in the space of germs of pair-mappings $(w, z)$ with zero values contains only finitely many different flag-contact orbits.

The set of dimensions $n, m$ will be called nice if pair-mappings whose germs at any point are simple (and hence stable with respect to a distinguished equivalence) form an open and dense subset in the space of pair-mappings of manifolds of these dimensions.

Again, $\Gamma^{+}$-nice dimensions are nice for flag-contact equivalence. The spaces of genotypes of all possible dimensions $r \leq m$ split into finite number of flag-contact orbits and a subset of codimension greater than $n$ in the germ space of pair-mapping with zero values.

In the following two sections we list all flag-contact simple germs. They happen to be weighted homogeneous. Hence (according to Remark 2 above) they are $R^{+}$-simple. Thus the sets of $\Gamma^{+}-, R^{+}$and flag-contact simple germs coincide. The same is true for the corresponding sets of nice dimensions.

Note that the list of flag-contact simple germs coincides (in complex category) with the list of Goryunov [8], [2] of simple functions on the complete intersections. Though the equivalence group are slightly different, this coincidence is also due to weighted homogeneity of the simple classes.

We are interested in the real classification and describe the list in full details.

Flag-contact equivalent pair-genotypes have contact equivalent genotypes of constraint mapping. Thus considering the well known [7] list of simple mapping germs (with respect to contact equivalence) and classifying for each of them the orbits of flag-contact equivalences which preserve this constraint germ $z$ and act on the space of germs functions $w$, one gets all candidates for simple pair-genotypes.

The following evident observations will be useful in the sequel:

1. The summation of $w$ with the function from $I_{z}$ does not change the flag-contact orbit of the pair. Really, such transformation corresponds to a shift diffeomorphism of the graph space $K_{0}$ which preserves the flag $K_{2} \subset K_{1} \subset K_{0}$. 
2. The tangent space $S T_{z}(w)$ to the orbit in the space of germs of $w$ of the subgroup $S F_{z}$ of flag-contact equivalences which preserve given germ $z$ is easy to calculate: $S T_{z}(w)=I_{z}+U_{z}(w)$, where $U_{z}(w)$ is a $C_{x}$-module of the derivatives of $w$ along vector fields $v$ such that $i_{v} d z_{j} \in I_{z}$ for all the components $z_{j}$ of $z$. Denote by $S V_{z}$ the module of these vector fields.

3. A genotype $(w, z)$ (which is a germ at some point $P$ of a reduced pair-mapping) will be called minimal if the restriction of the function $w$ to the zero-level set of the constraint mapping $z$ has minimum at this point $P$.

Evidently, only collections of minimal genotypes form multigerms corresponding to singularities of relative minima function.

\section{Classification, case $n=m$}

0. If rank of $g$ is $m$, all pairs $(f, g)$ are obviously equivalent to $(0, x), x \in \mathbf{R}^{m}$.

1. If rank of $g$ is $m-1$, the genotype is a pair of germs at the origin of functions $(w(x), z(x))$ in one variable. This space splits into the following set of flag-contact orbits

$$
B_{k, s}: w= \pm x^{s}, z=x^{k}, \quad k \geq 2, \quad k \geq s .
$$

For odd $s$ or for $s=k$, classes corresponding to \pm are equivalent. The codimension of the $B_{k, s}$ orbit equals $k+s-2$ in the space of germs at the origin of two functions in one variable.

2. If corank of $g$ is two then the genotype of $g$ is a set of two functions $g_{1}, g_{2}$ in two variables (denote them $x, y$ ) with zero 1 -jet.

The contact classification of two-jets of $g_{1}, g_{2}$ is the following (see also [7]):

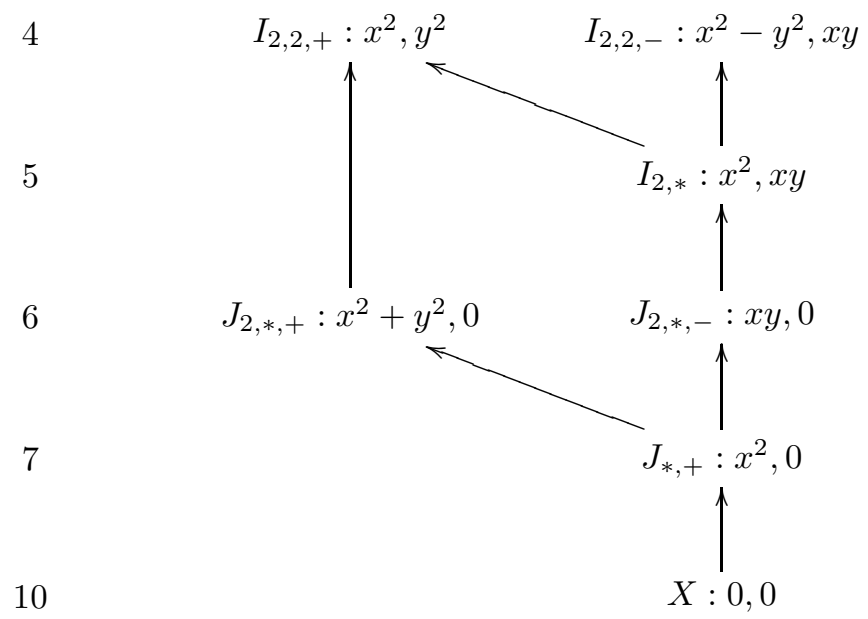

The codimensions of the classes in the space of two-jets with zero values at the origin compose the left column.

Two-jets $I_{2,2, \pm}$ are sufficient. 
Proposition 5. All pairs $(w, z)$ with $z \in I_{2,2, \pm}$ are simple. They are equivalent to pairs with one of the following functions $w$ :

- for $z \in I_{2,2,+}$ :

$$
\begin{gathered}
X_{4}^{+}: x+y ; \quad X_{5}^{+}: x ; \quad X_{6}^{+}: x y ; \quad X_{7}^{+}: 0, \\
X_{4}^{+} \longleftarrow X_{5}^{+} \longleftarrow X_{6}^{+} \longleftarrow X_{7}^{+},
\end{gathered}
$$

- for $z \in I_{2,2,-}$ :

$$
\begin{gathered}
X_{4}^{-}: x ; \quad X_{6}^{-}: \pm\left(x^{2}+y^{2}\right) ; \quad X_{7}^{-}: 0, \\
X_{4}^{-} \longleftarrow X_{6}^{-} \longleftarrow X_{7}^{-} .
\end{gathered}
$$

Proof. For $z \in I_{2,2,+}$ consider the factor-module $Q_{z}=C_{x} / I_{z}=\mathbf{R}\{1, x, y, x y\}$ and denote by $[w]$ the class in $Q_{z}$ of $w$. We have $S V_{z}=C_{x}\left\{x \frac{\partial}{\partial x} ; y \frac{\partial}{\partial y}\right\}$. If $j^{1}[w]=a x+b y$ and both coefficients $a, b$ are not zero then $S T_{z}(w)$ coincides with the whole maximal ideal in $C_{x}$. Remind that (due to the definition of flag-contact group) we consider only orbits of $w$ germs with $w(0)=0$. So, the germs with $a \neq 0, b \neq 0$ form one orbit $X_{4}^{+}$.

The orbit $X_{5}^{+}$contains all $w$ such that only one of the coefficients $a, b$ vanishes.

If $j^{1}[w]=0$ but $j^{2}[w] \neq 0$ then $w \in X_{6}^{+}$. The germs $w \in I_{z}$ form the orbit $X_{7}^{+}$.

The case $z \in I_{2,2,-}$ is treated similarly.

Germs with two-jets from class $I_{2, *}$ split into a series of (simple) contact orbits $I_{2, k}$ : $x^{2} \pm y^{k}, x y, k \geq 3$. (For odd $k$ signs \pm correspond to the same orbit.)

Proposition 6. All pairs $(f, g)$ with $g \in I_{2,3}$ are simple. They are equivalent to pairs with one of the following functions $f$ :

$$
\begin{gathered}
Y_{5}: y ; \quad Z_{6}: x ; \quad Z_{7}: y^{2} ; \quad Z_{8}: y^{3} ; \quad Z_{9}: 0, \\
Y_{5} \longleftarrow Z_{6} \longleftarrow Z_{7} \longleftarrow Z_{8} \longleftarrow Z_{9} .
\end{gathered}
$$

Proof. For $z \in I_{2,3}$ the factor-module $Q_{z}=C_{x} / I_{z}$ is generated over $\mathbf{R}$ by classes of $1, y, x, y^{2}, y^{3}$. The weights $\frac{1}{2}$ and $\frac{1}{3}$ of $x$ and $y$ define weighted gradings on $Q_{z}$ and $S V_{z}=C_{x}\left\{x \frac{\partial}{\partial x}+y \frac{\partial}{\partial y} ;-2 y^{2} \frac{\partial}{\partial x}+3 x \frac{\partial}{\partial y}\right\}$. If the lowest degree of non-zero terms in $[w]$ is $\alpha$ then the space $S T_{z}(w) \subset Q_{z}$ contains all germs of degree $\geq \alpha$. This proves the proposition.

Proposition 7. Pairs with $z \in I_{2, k}$ for $k \geq 4$ are simple if and only if the linear form of $w$ is non-degenerate $\left(\frac{\partial w}{\partial y} \neq 0\right)$. If so, then the pair is equivalent to the normal form $Y_{2, k}: f=y, g=\left(x^{2} \pm y^{k}, x y\right)$. If not, then the germ is adjacent to the lowest non-simple class, which has codimension 7 and consists of pairs $w=x+a y^{2}, z=\left(x^{2} \pm y^{4}, x y\right) \in I_{2,4}$, $a \in \mathbf{R}$.

Proof. Weights $\frac{1}{2}$ and $\frac{1}{k}$ of $x$ and $y$ define a grading on $Q_{z}$ for $z \in I_{2, k}$. The lowest non-zero term of it is the class of $[y]$. Since the $\frac{\partial}{\partial y}$-components of vector fields from $S V_{z}=C_{x}\left\{x \frac{\partial}{\partial x}+y \frac{\partial}{\partial y} ;-2 y^{k-1} \frac{\partial}{\partial x}+k x \frac{\partial}{\partial y}\right\}$ form total space $C_{x}$, all $w$ germs with non-zero $y$ terms are equivalent.

If $k=4$ there exists a two-dimensional subspace $W_{\frac{1}{2}}$ of $Q_{z}$ of degree $\frac{1}{2}$ generated by $x, y^{2}$. Consider the germ $w$ with zero coefficient of lower degree term $[y]$. The intersection 
of $S T_{z}(w)$ with $W_{\frac{1}{2}}$ is at most one-dimensional, since there is only one (up to the multiplication by a constant) vector field of degree 0 - the Euler vector field. Thus, $W_{\frac{1}{2}}$ splits into a one-parameter family of orbits, which, therefore, fail to be simple. The genotypes with $z \in I_{2, k}, k>4, \frac{\partial w}{\partial y}=0$ are adjacent to this non-simple class.

Proposition 8. Pairs with two-jet of $g$ from the class $J_{2, k}$ are non-simple. They are adjacent to non-simple classes with constraint mapping g equivalent to $\left(x y, x^{3}+y^{3}\right)$ or to $\left(x^{2}+y^{2}, x^{3}+y^{3}\right)$. The codimension of the latter classes equals 6 .

Pr o of. The stationary algebras $S V_{z}$ in these cases contain only one (Eulerian) vector field of homogeneous degree 0 , while the space of 1-jets of classes from $Q_{Z}$ is twodimensional. Thus, 1-jets of $w$ are not simple.

3. There are no simple genotypes with rank of $g$ less than $m-2$. Even there are no contact simple germs of such mappings $g([7])$. Each of them is adjacent to the germ with genotype whose 2-jet is a collection of three quadratic forms in three variables. The dimension of this space is 18 , the contact equivalences acts on two-jets by the product of linear groups in the source and the target of the dimensions $9+9$. Since the stationary subgroup of every such 2-jet is at least 1-dimensional (it contains products of homogeneous scalings by $\lambda$ and $\lambda^{-1}$ in the source and the target), the space of orbits is not discrete.

Remarks.

1. All simple and also "fencing" non-simple genotypes which we have classified in this section are minimal. In each case the zero-level set of $z$ consist only of one point-the origin.

2. The graphs of minima functions for fencing non-simple classes are non-simple. The values of moduli have simple geometrical meaning.

\section{Classification, case $n>m$}

0. If $g$ has full rank (equal to $m$ ) then the flag-contact classification of corresponding pairs transforms into that of contact orbit of genotypes of $f$, being the functions on $n-m$ variables. Thus, the list of simple classes is standard $\mathrm{A}, \mathrm{D}, \mathrm{E}$ list. If $n-m \geq 3$, the first non-simple class (corresponding to parabolic singularity $P_{8}$ ) has codimension 6 . However, the germs of this class have no minimum at the origin. The lowest codimension of the non-simple class with non-empty relative minima function germ is 7 and is achieved on the stratum of the parabolic singularity $X_{9}$ of functions in two variables. It represents the lowest codimension of the non-simple class for $n-m=2$. If $n-m=1$ all (except the subset of infinite codimension) germs belong to simple classes $A_{k}$.

1. If the corank of $g$ is 1 , the genotype is a pair of functions $(w, z)$ in $s=n-m+1$ variables.

If $s>2$, all such pairs with zero linear part of $w$ are non-simple. Really, they are adjacent to genotype with $s=3$. Consider the 12-dimensional space of two-jets of them. The flag-contact action on this space coincides with the action of the product of the linear group of the source space (of dimension 9) and the three-dimensional triangle subgroup 
of the linear group in the target. Since the stationary subgroup for any genotype contains opposite scalings in source and target, the orbit space is not discrete. The codimension of this set of non-simple pairs is 6. Its stratum of this codimension is formed by oneparameter families of orbits $Z_{6}^{e}: w= \pm x_{1}^{2}+\beta x_{2}^{2}, z=x_{1}^{2}+x_{2}^{2}+x_{3}^{2}$ and $Z_{6}^{h}: w=x_{1}^{2}+\gamma x_{2}^{2}$, $z=x_{1} x_{2}+x_{3}^{2}, \beta, \gamma \in \mathbf{R}$. Note that these genotypes are minimal (for all $\beta$ and $\gamma \geq 0$ ).

Consider then the case when $w$ has a non-trivial linear part. Reduce $w$ to the form $w=x_{1}$ by an appropriate change of the variables in the source space.

Proposition 9. Any simple genotype of this type is flag-contact equivalent to the genotype $\left(x_{1}, z\right)$, where the germ of $z$ is one of the following list (which corresponds to the list of simple boundary singularities):

$B_{k}: x_{1}^{k} \pm x_{2}^{2} \pm \ldots \pm x_{s}^{2} ; \quad C_{k}: x_{1} x_{2}+x_{2}^{k-1} \pm x_{3}^{2} \pm \ldots \pm x_{s}^{2} ; \quad F_{4}: x_{1}^{2}+x_{2}^{3} \pm x_{3}^{2} \pm \ldots \pm x_{s}^{2}$.

The codimension of these classes equals $k+s-2$.

R e m a r k. The codimension of the first non-simple class of orbits (corresponding to boundary singularity $F_{5}^{*}: z=x_{1}^{2} \pm x_{2}^{4}$ ) equals 5 . For sign + this genotype is minimal.

Proof. Having reduced the $w$-germ to normal form, we normalise now $z$ by the equivalences which preserve $w$. The tangent space at $z$ to the orbit of such subgroup is the set $T_{z}$ of the functions $\phi z+i_{v} d z$ with arbitrary $\phi$ and with vector field $v$ satisfying the condition $i_{v} d x_{1} \in C_{x}\left\{z, x_{1}\right\}$. Thus the space $T_{h}$ coincides completely with the tangent space to the orbit of the germ $h$ with respect to the (right) changes of variables $x$ which preserve the hyperplane $x_{1}=0$, and multiplications of $h$ by arbitrary non-zero function.

Hence, the flag-contact orbits coincide exactly in this case with the contact classes of boundary singularities [1], [2] and the result follows.

R e mark. Only genotypes $B_{k}: x_{1}^{k}-x_{2}^{2}-\ldots-x_{s}^{2}$ are minimal. The relative minima functions of the stable germ with these genotypes do not depend of numbers of variables of its quadratic part. When $s=1$ (that is, $n=m$ ), we get the relative minima function of genotype $B_{k, 1}$.

This coincidence provides certain stabilisation of $B_{k}$ singularities in the lists of simple classes with growing up difference $n-m$.

In the remaining case, when $s=2$ and one-jet of $f$ vanishes, denote by $(x, y)$ the coordinates in the source space of genotype $\mathbf{R}^{2}$.

Proposition 10. All simple genotypes $(w(x, y), z(x, y))$ with $s=2$ and $j^{1} w=0$ are the following:

$$
\begin{gathered}
C_{k, l}^{-}: z=x y, w=x^{k} \pm y^{l}, \quad k, l \geq 2 ; \\
C_{k, k}^{+}: z=x^{2}+y^{2}, w=x^{k}, \quad k \geq 2 ; \\
F_{2 k+1}: z=x^{2}+y^{3}, w= \pm y^{k}, \quad k \geq 2 ; \\
F_{2 k+2}: z=x^{2}+y^{3}, w=x y^{k}, \quad k \geq 1 .
\end{gathered}
$$

Remarks.

1. For odd $k, l$ signs \pm correspond to equivalent classes. The codimensions of $C_{k, l}^{-}$is $k+l$, that of $C_{k}^{+}$is $2 k$ and the codimension of $F_{i}$ is $i-1$. 
2. Among these genotypes only

$$
\begin{aligned}
C_{2 k, 2 l}^{-} & : z=x y, w=x^{2 k}+y^{2 l}, \quad k, l \geq 1 ; \\
C_{k, k}^{+} & : z=x^{2}+y^{2}, w=x^{k}, \quad k \geq 2 ; \\
F_{4 k+3} & : z=x^{2}+y^{3}, w=-y^{2 k+1}, \quad k \geq 1 ; \\
F_{4 k+1} & : z=x^{2}+y^{3}, w=y^{2 k}, \quad k \geq 1
\end{aligned}
$$

are minimal.

P r o of. The adjacency diagram of contact classes of functions $z$ starts by the sequence $A_{1}^{ \pm} \longleftarrow A_{2} \longleftarrow A_{3}^{ \pm} \ldots$, where

$$
A_{1}^{-}: x y ; \quad A_{1}^{+}: x^{2}+y^{2} ; \quad A_{2}: x^{2}+y^{3} ; \quad A_{3}^{ \pm}: x^{2} \pm y^{4} ; \quad \ldots .
$$

The stationary algebra of $z \in A_{1}^{-}$is generated over $C_{x, y}$ by two vector fields: $e_{0}=$ $x \frac{\partial}{\partial x}+y \frac{\partial}{\partial y}$ and $e_{1}=x \frac{\partial}{\partial x}-y \frac{\partial}{\partial y}$. Subtraction from function $w$ a germ $u \in I_{z}$ does not change the flag-contact class of the pair. Thus we may assume $z$ to have the form $z=f_{1}(x)+f_{2}(y)$ without any term divisible by $x y$. The lowest degrees $k, l$ of $x$ and $y$ with non-zero coefficients in Taylor series for these two functions determine the orbit.

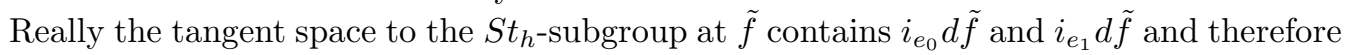
contains monomials $x^{k}$ and $y^{l}$. This proves the first line of the list.

The remaining cases are studied analogically. The non-simple class of lowest codimension $(=6)$ consists of pair-genotypes with $z \in A_{3}, j^{1} w=0$ and is adjacent to non-simple class $F_{5}^{*}$.

2. There are no simple genotypes if the corank of $g$ is 2 or more. Really, all such genotypes (which form a subset of codimension $2(n-m+2) \geq 6$ ) are adjacent to the genotype of corank 2 , which consists of a germ of a function $f$ in three variables and of germs of two components $g_{1}, g_{2}$ (without linear terms) of the constraint mapping $g$.

Denote by $L$ the 15 -dimensional space of two-jets of $g_{1}, g_{2}$ and one-jet of $f$. The flag-contact equivalences induce the action on this space, which is the product of linear transformations in the source (which form 9-dimensional group), and the linear transformations in the target, which preserve the projection $\mathbf{R}^{3} \rightarrow \mathbf{R}^{2},\left(f, g_{1}, g_{2}\right) \mapsto\left(g_{1}, g_{2}\right)$. Since one-jets of $g_{i}$ vanish, the transformations in the targets acts on $L$ as block-diagonal matrices, forming the group of dimension 5. Thus the dimension of the group is less than the dimension of the space and the orbits form continuous families of non-equivalent jets.

R e mark. The fencing non-simple minimal classes, enumerated in this section, also produce non-simple relative minima function germs.

7. Proofs of Theorems 1-3. Theorems 1 and 2 are immediate consequences of the transversality theorem and the classification of flag-contact minimal simple genotypes. As it was already mentioned, the fencing non-simple (and minimal) flag-contact equivalence classes produce non-simple singularities of relative minima function.

To prove Theorem 3, besides the classification of simple minimal orbits, we have to describe normal forms of some non-simple flag-contact classes. Namely, there is one 
stratum (of codimension 5) of unimodal singularities $F_{5}^{*}$ in the space of pair-mapping germs $(f, g): \mathbf{R}^{5}, 0 \rightarrow \mathbf{R} \times \mathbf{R}^{4}, 0$.

Also there is a stratum $Z_{6}^{e, h}$ of codimension 6 in the space of pair-mapping germs $(f, g): \mathbf{R}^{6}, 0 \rightarrow \mathbf{R} \times \mathbf{R}^{4}, 0$.

Note that these singularities $F_{5}^{*}, Z_{6}^{e, h}$ are weighted homogeneous, and therefore flagcontact orbits coincide with $R^{+}$- ones.

According to the transversality theorem, the pair-mappings with jet-extensions transversal to these strata (but not to the orbits) are generic.

Consider such a pair-mapping $(f, g)$ and corresponding adapted coordinates $x, y$ on $\mathbf{R}^{n}, 0, x \in \mathbf{R}^{n-r}, y \in \mathbf{R}^{r}$ and $z, u$ on $\mathbf{R}^{4}, 0, z \in \mathbf{R}^{m-r}, u \in \mathbf{R}^{r}$, such that the constraint mapping $g$ takes the form $\tilde{g}:(x, y) \mapsto(z(x, y), u)$ with $u=y$ and the components $z_{i}(x, 0)$ belong to the square of the maximal ideal of the ring $C_{x}$ of germs at the origin of functions in $x$. Here $r$ is the rank of $g$.

The genotype $(w, z)$ of the pair-mapping is defined up to the action of flag-contact group. The jet-extension of pair-mapping in adapted coordinates $(\tilde{f}, \tilde{g})$ rests, of course, transversal to the stratum, which is one-parameter family of flag-contact orbits of genotypes.

Associate to such a pair-mapping $(\tilde{f}, \tilde{g}): \mathbf{R}^{n} \rightarrow \mathbf{R} \times \mathbf{R}^{m}$ its suspension $(\breve{f}, \breve{g})$ : $\mathbf{R}^{n} \times \mathbf{R} \rightarrow \mathbf{R} \times \mathbf{R}^{m} \times \mathbf{R}$,

$$
(\breve{f}, \breve{g}):(x, y, \lambda) \mapsto(\tilde{f}(x, y)+\lambda \xi(x), \tilde{g}(x, y)+\chi(x) \lambda, y, \lambda),
$$

the jet-extension of which is transversal to flag-contact orbit of the genotype. Thus $(\breve{f}, \breve{g})$ is stable and is equivalent to standard versal deformation of the genotype. The equivalence diffeomorphism of the target space might be taken in a special form (respecting the adapted coordinates fibration):

$$
\theta_{2}:(z, u, \lambda) \mapsto(Z(z, u, \lambda), U(u, \lambda)) .
$$

Thus the initial pair is equivalent to the restriction of the standard versal deformation of the genotype to hypersurface $\lambda=0$. The equation of it in variables $U$ might be written in the form $U_{\mu}=\varphi\left(U_{1}, \ldots, U_{\mu-1}\right)$, where $U_{1}, \ldots, U_{\mu-1}$ are parameters on the transversal to the stratum and $U_{\mu}$ is a parameter of versal deformation along the stratum in transversal direction to the orbit. The function $\varphi$ defines a functional modulus (with respect to $\Gamma^{+}$-equivalence) of the corresponding non-simple and non-stable normal form.

\section{References}

[1] V. I. Arnol'd, S. M. Guseı̆n-Zade, A. N. Varchenko, Singularities of Differentiable Maps I, Monogr. Math. 82, Birkhäuser, Boston, 1985.

[2] V. I. Arnol' d, V. V. Goryunov, O. V. Lyashko and V. A. Vasil'ev, Singularity theory. II. Classification and applications, in: Dynamical Systems VIII, Encyclopaedia Math. Sci. 39, Springer, Berlin, 1993, 1-235.

[3] L. N. Bryzgalova, The maximum functions of a family of functions that depend on parameters (in Russian), Funktsional. Anal. i Prilozhen. 12 (1978), no. 1, 66-67; English transl.: Functional Anal. Appl. 12 (1978), 50-51. 
[4] J. Damon, The unfolding and determinacy theorems for subgroups of $\mathcal{A}$ and $\mathcal{K}, \mathrm{Mem}$. Amer. Math. Soc. 50 (1984), no. 306.

[5] A. A. Davydov, Singularities of the maximum function over a preimage, in: Geometry in Nonlinear Control and Differential Inclusions, Banach Center Publ. 32, Warsaw, 1995, $167-181$.

[6] A. A. Davydov, V. M. Zakalyukin, Point singularities of relative minimum on three dimensional manifold (in Russian), Trudy Mat. Inst. Steklov. 220 (1998), 113-129; English transl.: Proc. Steklov Inst. Mat. 220 (1998), 109-125.

[7] M. Giusti, Classification des singularités isolées d'intersections complètes simples, C. R. Acad. Sci. Paris Sér. A-B 284 (1977), A167-A170.

[8] V. V. Goryunov, Geometry of the bifurcation diagrams of simple projections onto a line (in Russian), Funktsional. Anal. i Prilozhen. 15 (1981), no. 2, 1-8; English transl.: Functional Anal. Appl. 15 (1981), 77-82.

[9] O. V. Lyashko, Classification of critical points of functions on a manifold with singular boundary (in Russian), Funktsional. Anal. i Prilozhen. 17 (1983), no. 3, 28-36; English transl.: Functional Anal. Appl. 17 (1983), 187-193.

[10] V. I. Matov, Topological classification of the germs of functions of the maximum and minimax of families of functions in general position (in Russian), Uspekhi Mat. Nauk 37 (1982), no. 4, 167-168; English transl.: Russian Math. Surveys 37 (1982), no. 4, 127-128.

[11] V. M. Zakalyukin, Envelopes of families of wave fronts and control theory (in Russian), Trudy Mat. Inst. Steklov. 209 (1995), 133-142; English transl.: Proc. Steklov Inst. Math. 209 (1995), 114-123.

[12] V. M. Zakalyukin, Singularities of circles contact with surfaces and flags, Funktsional. Anal. i Prilozhen. 31 (1997), no. 2, 73-76; English transl.: Functional Anal. Appl. 31 (1997), $131-133$ 